make satisfactory experiments on the influx, efflux, and varying density of mosquitos without such an analysis, and one, I may add, far more minute than has been attempted here. The subject has suffered much at the hands of those who have attempted experiments without adequate preliminary consideration, and whose opinions or results have seriously impeded the obviously useful and practical sanitary policy referred to. The statement, so frequently made, that local antipropagation measures must always be useless owing to immigration from outside is equivalent to saying that the population of the United States would remain the same even if the birth-rate were to be reduced to zero. In a recent experiment at Mian Mir in India the astounding result was obtained that the mosquito density was, if anything, increased by the antipropagation measures, which is equivalent to saying that the population of the United States would be increased by the abolition of the birth-rate. In the meantime, I for one, must continue to believe the somewhat selfevident theory that antipropagation measures must always reduce the mosquito density, even if the results at Havana, Ismailia, Klang, Port Swettenham, and other places are not accepted as irrefragable experimental proof of it.

\section{THE CAUSE AND TREATMENT OF PRURITUS ANI}

Bx F. C. WALLIS, M.B., F.R.C.S.,

Surgeon, Charing Cross Hospital, St. Mark's Hospital, and Grosvenor Hospital for Women and Chil dren.

The papers recently published in the British Mrdical JOURNAI on this subject are interesting in many ways, but especially because they show that the cause, the real cause of pruritus ani-that is inveterate pruritus ani, as Sir Charles Ball properly calls it-is still not known, or not recognized.

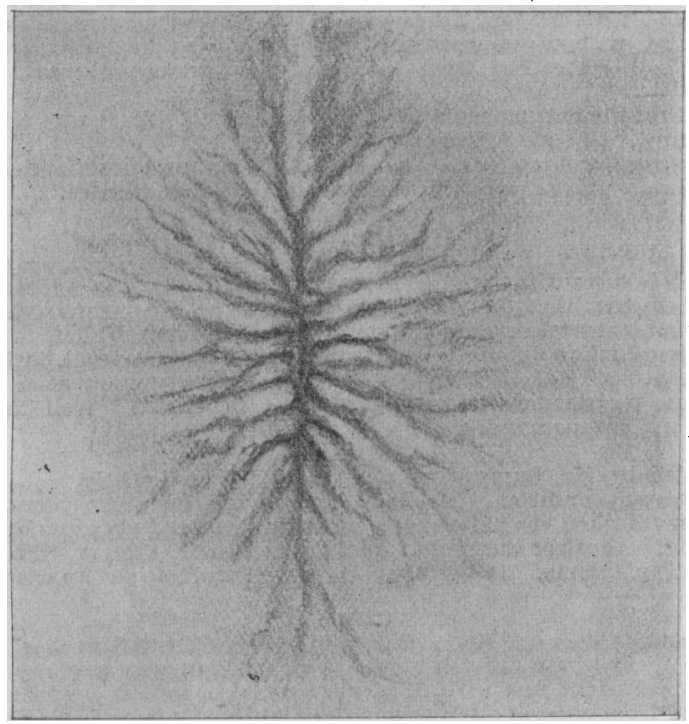

Fig. $x$.

In 1897 , in the St. Bartholomew's Hospital Journal, and again in 1902, in the Clinical Journal, I published the results of my investigations concerning this troublesome complaint. To my own experience I can now add that of one or two others ; and, as the united experience is both sufficient and satisfactory, it will, I think, be useful to repeat what I have already written.

\section{Pathologr.}

First let me say that these remarks do not apply to passing anal irritation, which may be due to one of the many assigned causes, and which will disappear when the cause, such as errors of diet, eczema, gout, thread-worms, etc., is treated. They apply to the inveterate cases, which go on year after year, in spite of all sorts of treatment-the constant irritation occurring night after night, bringing in its train other symptoms due to want of sleep, such as inability to work, irritability, dyspepsia, loss of appetite and loss of weight, making life a veritable burden.

The local appearance of the skin area affected by pruritus ani is quite typical (as in Fig. I), and should at once be recognized as pathognomonic of the trouble. Chronic irritation and constant scratching produce a chronic inflammation of the skin resulting in a complete alteration in the character of the integument, which becomes largely infiltrated with fibrous tissne, loses its elasticity, and becomes covered with dead and often sodden epithelium.

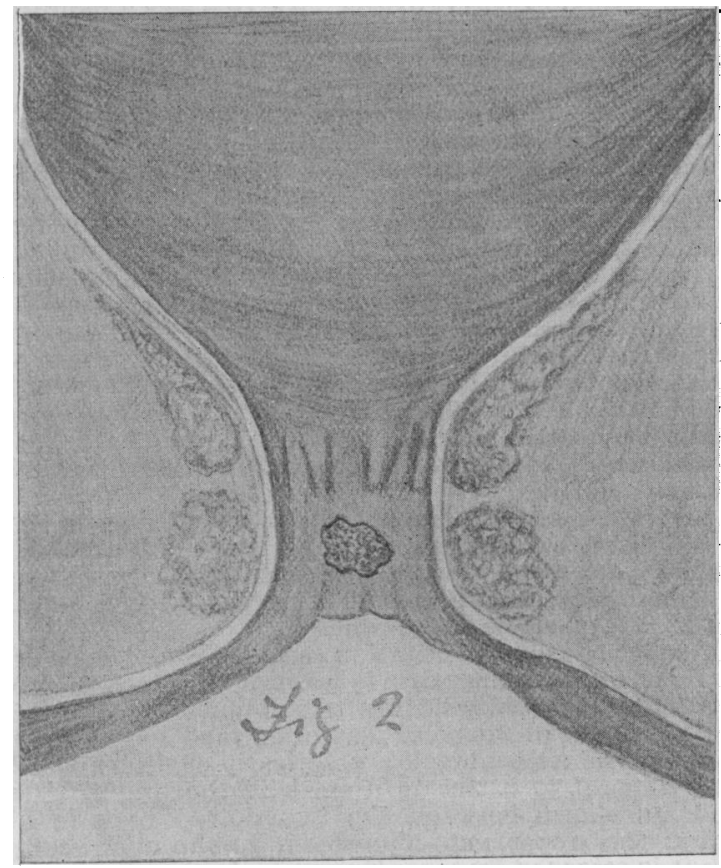

Fig. 2.

In over 90 per cent. of cases of pruritus ani which I have In a st. Mark's I have found a shallow ulcer situated between the two sphincters (Fig. 2), mole often in the posterior segment than in the anterior, and generally near the dorgal midline. In some cases there is more than one ulcer, and again in others there are various clefts which almost or entirely surround the bowel (Fig. 3).

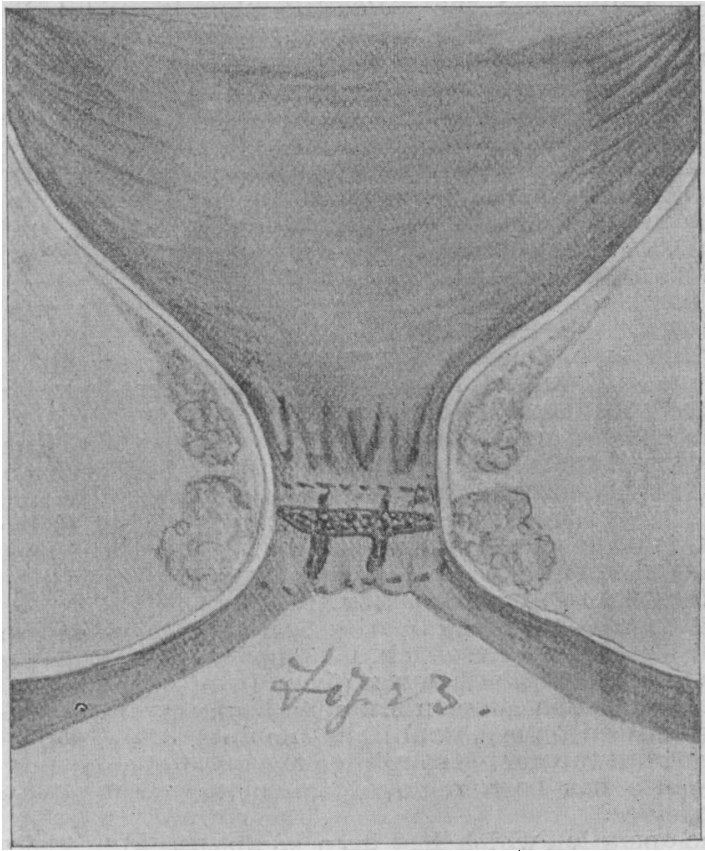

Fig. 3.

The method of fusion of the proctodeum with the blind end of the gut is, I believe, the cause of this frequent ulcer. The fusion takes place at a variable line between the sphincters. The lining membrane of the proctodeum is thin, and, unlike 
mucous membrane, is scantils supplied with blood vessels, and thus abrasions in this locality are easily brought about. But because of the ever-altering condition of the sphincter, and because of the contents of the bowel, they rarely heal of their own accord. The abrasion becomes an ulcer covered with chronic granulation tissue, exuding an irritating secretion, which causes pruritus. As this was a definite and constant lesion, I determined to treat it, believing it to be the cause of this distressing complaint.

Treatment.

Without going over various details of early cases, I may say that the results ar $\gg$ much better when patients can lie up for ten to fourteen days, when the following treatment is carried out : The usual preparation for a rectal operation having been carried out, the patient is anaesthetized, and put in the lithotomy position. The sphincter is moderately stretched and the ulcer or ulcers brought into view and treated with the electric thermo-cautery, and the cautery is also applied to the thickened skin as well, especially in any cases where fissures or clefts exist between the hypertrophied skin folds. Vaseline is applied to the cauterized area, and a morphine suppository inserted into the bowel. A pad of wool is kept in position by a T-bandage, and the patient is put back to bed and kept there.

A purge is given on the third night and a warm boracic bath is taken twice a day. After the bath the skin is thoroughly dried and powdered with starch and zinc powder, and a small piece of cotton-wool impregnated with powder is introduced just inside the sphincter.

In all these cases the irritation ceases either at once or after a few days, and if proper care is taken it does not return, and the patients are usually well in about fourteen days. but the absolute healing of the ulcer may often take longer than this. In cases where the abrasion practically encircles the bowel I have thought it better to dissect off the ring of tissue involved, as shown between the two dotted lines in Fig. 3 , bringing the upper cut edge down to the anal margin, to which it is attached by a continuous catgut suture-thus re moving all the lining membrane of the proctodeum. These cases are not so immediately successful as the others because the condition of the muco-cutaneous margin is indifferent and healing is at times protracted, and some temporary contraction may occur, but with proper care this disappears, the result is good and the pruritus is cured.

Having regard to the large number of out-patients suffering from this trouble, it is obviously impossible to take them all into hospital, and the following plan is adopted. The patient being placed in the knee-elbow position, a bivalve speculum is inserted into the rectum and kept in position about half opened. Some eucaine is injected behind the alcer. This renders the area anaesthetic and brings the ulcer into prominence. The ulcer is either treated with lactic acid or burnt with the electric thermo-cautery, the speculum removed, and a morphine suppository introduced. Zinc and starch powder is dusted over the skin and a pad and T-bandage are applied. The subsequent treatment is the same as has already been described.

A large percentage of these cases are cured; many are improved. A few do not respond for long to the treatment, but this is because it is not properly carried out by the patients, and, indeed, it must be of ten a matter of considerable difficulty for them to do so.

The interesting feature in many cases is the immediate cessation of the irritation after the cautery has been applied. This is well shown in the accompanying cases. The irritation may recur spasmodically to a slight extent, but it is easily allayed and soon disappears entirely. The permanent success of the treatment depends largely upon carefal nursing under the personal supervision of the operator.

The suggestion by Sir Charles Ball that these cases are of central origin is one which I cannot accept without further evidence than that which was given in his interesting paper published in the BRITIsH MrDICAL JoURNAL of January $218 t$. Long-continued peripheral irritation does, of course, produce a change in the nerves supplying the affected area, but when the cause has been removed the nerves gradually recover themselves.

The operation which has been so successful in Sir Charles Ball's hands is not one which I should like to see made practice of. I do not think it is necessary, and in many cases it will end in disaster.

The following analysis of 272 cases seen at St. Mark's Hospital will show how frequently the ulcer occurs:

\begin{tabular}{|c|c|c|c|c|c|}
\hline $\begin{array}{l}\text { 1. Ulcer record } \\
\text { 2. Ulerer record } \\
\text { 3. Cases not inv } \\
\text { 4. Cases associa }\end{array}$ & $\begin{array}{l}3 \text { pr } \\
\text { p de } \\
\text { gate } \\
\text { with }\end{array}$ & $\begin{array}{l}\text { sent } \\
\text { initely } \\
\text { d for ul } \\
\text { haemo } \\
\text { fistula }\end{array}$ & & $\begin{array}{c}\cdots \\
\ldots \\
\cdots \\
\ldots\end{array}$ & $\begin{array}{r}140 \\
2 \\
87 \\
22 \\
8\end{array}$ \\
\hline $\begin{array}{ll}\text { 5. } & \quad "\end{array}$ & ", & $\begin{array}{c}\text { fistula } \\
\text { for }\end{array}$ & ... & ... & \\
\hline 7. Other causes & & $\begin{array}{l}\text { nissure } \\
\quad . .\end{array}$ & $\cdots$ & ... & \\
\hline 10 dat & & $\ldots$ & ... & & 72 \\
\hline
\end{tabular}

Of the 87 cases not investigated many were of a trivial character, but it is reasonable to suppose that an ulcer would have been found in a fair proportion of this number if it had been looked for.

The notes of the following cases have been taken from the practices of Mr. A. Chune Fletcher and Mr. H. M. Ramsay, as well as from my own, and illustrate the beneficial results which have been obtained in a large number of cases.

Examples of Cazes Treated by the Cautery, or Applications of Lactic Acid, or by Both.

$$
\text { CASE I. }
$$

Male, aged 45, gouty. Has had pruritus ani for years. Operated on previously for piles, without relief of pruritus. Suffers from insomnia and general malaise.

An ulcer was seen in the posterior segment and cauterized under an anaesthetic.

After-treatment and Result.-Rest for ten days; boracic baths; zinc oxide and starch powder. Seen after some months: looked five years younger, had put on flesh, slept well, no pruritus. The treatment was carried out in r9oo, and there has been no recurrence.

CASE II.

Male, aged 43, highly neurotic. Pruritus ani for years. Had had two operations, but with no good effect. Ulcer discovered and cauterized. After-treatment as in Case I. Result: Pruritus ani gradually disappeared, and patient's general health greatly improved.

\section{CASE IIr.}

Under the care of Mr. H. M. Ramsay. Male, aged 22. Pruritus ani associated with eczema for five jears. Operated on for piles three years ago, without improvement. The condition kept him awake at night; he had lost flesh, and was in a low, nervous condition.

Operation February, 1904, under an anaesthetic. A posterior ulcer just within the external sphincter was cauterized. Slept well all night. no itching. In bed fourteen days.

After-treatment as in Case I. Result : Pruritus cured and eczema cleared up. Put on weight, in better health, no recurrence.

CASE IV

Treated by Mr. Ramsay. Male, aged 53, seen in June, r904. Had suffered from pruritus ani for " years." There was also a local condition of eczema. Complained of sleeplessness due to constant soratching. Had tried many treatments with only slight temporary relief.

On examination an ulcer was found on the anterior wall, just inside the sphincter. This was treated by the electric cautery after a local injection of eucaine. After-treatment as in Case 1 . Well in three weeks, and no recurrence.

Case v.

Treated by Mr. Ramsay. Male patient. Seen in July, rgo4. In a low, nervous condition. Has had pruritus ani for some years, which commences when the patient gets warm in bed, and prevents him from sleeping. An ulcer was found and treated as in Case IV, with entiro relief of symptoms. The patient sleeps well and has put on weight.

\section{CASE VI.}

Under the care of Mr. A. Chune Fletcher. Treated in r898. Male, aged 32. Pruritus ani for seven years. There was a gouty family history. Patient was in a low, nervous, despondent condition, and suffered from want of sleep due to the nightly-recurring irritation.

The anal skin showed the usual parchment-like condition, and was chafed and fissured. A considerable ulcer was found on the posterior wall between the sphincters, and was treated by the application of lactic acid. The itching was checked at once, and, although the nlcer took some time to heal, his local and general condition rapidly improved, and he made a complete recovery.

CASE VII.

Treated by Mr. Fletcher. Male, aged 42 . Pruritus ani and sense of "twitching" in rectum for three years. Posterior and anterior ulcers. Treated by lactic acid and cautery, with external hot douching at night, and "fuller's earth" to affected skin. Irritation ceased almost immediately, but ulcers took nearly three months to heal.

$$
\text { CASE VIII. }
$$

Treated by Mr. Fletcher. Male, aged 42. Pruritus ani for two years. Posterior ulcer, treated by bi-weekly applications of lactic acid and daily injections of hazeline and water equal parts. Well in three weeks.

Treated by Mr. Fletcher. Female, aged 40. Pruritus ani, varying greatly in intensity. Large posterior and sthall anterior nlcers. Treatment : Lactic acid, hazeline and water, cautery to edges of ulcer. Well in six weeks. 
Case $x$.

Treated by Mr. Fletcher. Male, aged 41. Much pruritus every night when warm in bed. After painless action of bowels, a feeling of burning comes on, with dull aching pain across the back and down the thighs, which varies in degree, but never quite goes. Posterior ulcer discovered. Lactic acid applied. Pruritus disappeared and other symptoms gradually lessened as ulcer healed. Well in one month.

Examples of Cases where the Whole Circumference of the Lining Membrane was Dissected Off.

CASE XI.

Female, aged about 40 , single ; seen November, 1902. Pruritus an for many years. Patient was in a thin, miserable, nervous condition unable to sleep on account of the irritation. Inside the external sphincter the whole circumference of the bowel was practically denuded of epithelium. The affected ring of tissue was dissected off, and the upper edge sewn to the skin. Healing was slow in this instance, but the condition of pruritus ani gradually disappeared. This patient developed a fissure nearly two years after, which was operated on in November, rgo4. No return of pruritus.

\section{CaSe XII.}

Male, aged 40. Pruritus ani for twenty-five years. Much worse of late. No treatment has done any good. Suffers from insomnia caused by itching. Typical external appearance. Inside the sphincter the entire circumference of the bowel was denuded of he sphincter the internal " pruritic area" was dissected off, the patient being anaesthetized, and the upper edge sewn to the skin margin. The condition of pruritus gradually disappeared, and when the patient left the home hree weeks later he was practically well of it.

Seen since and says he is a different man. Sleeps well and eats well. There was some slight contraction of the orifice, but this was easily got rid of by using a conical vulcanite bougie for a few times.

\section{Case XIII.}

Male, aged 28. Pruritus ani for years. The whole circumference of the lining membrane was affected, this was dissected off and the upper edge sewn to the skin margin. Well in three weeks.

\section{CaSe XIv.}

Female, aged 54. Pruritus ani for three years. Skin around anus excoriated, white and sodden. Ulcers found laterally and posteriorly. Membrane dissected off. Well in three weeks.

\section{CASE XV.}

Male, aged 54. Pruritus ani for years. Skin around anus white and sodden-looking. Ulcers found. Ring of membrane dissected off. Well in five weeks.

\section{THE SURGICAL ANATOMY AND OPERATIVE TREATMENT OF TUBERCULOUS GLANDS OF NECK.}

BY W. GREEN WOOD SUTCLIFFE, F.R.C.S.ENG., Surgeon, Royal Sea-Bathing Hospital ; Metropolitan Asylums Board Children's Home; and Victoria Home for Invalid Children, Margate.

THE anatomy of the lymphatic glands of the neck has been very thoroughly described by Leaf in his Surgical Anatomy of the Lymphatic Glands, in Poirier and Cunéo's Lymphatics, translated by Leaf, and is also effectively treated in Cunningham's Textbook of Anatomy, p. 865, where a sufficiently full description of the normal glands is to be found. The practical surgeon has, however, to deal with structures that are no longer normal, and a thoroughly intimate knowledge of the anatomy of the neck as distorted by masses of enlarged glands is only to be acquired by a large operative experience. For while with small superficial glands the only practical point is to avoid scarring the skin in a wrong direction, a really extensive case involves the exposure of almost the entire contents of both the anatomical triangles, and structures as widely separated as the facial nerve and the thoracic duct may require special attention to avoid their injury or actual division.

ANatomy.

For descriptive purposes the cervical glands may be divided into superficial and deep groups, including in the latter all glands beneath the layer of cervical fascia that forms the sheath of the sterno-mastoid. The superficial groups again may be divided into three sets-superficial parotid, superficial submaxillary, and superficial cervical.

1. The superficial parotid, two or three in number, are, when taberculous, almost always found to be broken down, and are imbedded in the salivary gland. I have never seen the facial nerve damaged by their removai, so that the greater part of the pes anserinus must be protected by a sufficient layer of salivary gland tissue; but the proximity of the nerve is not likely to be forgotten, and most operators will keep exceedingly close to the capsule of the gland, and, if it be adherent, scrape rather than dissect.

2. The superficial submaxillary are among the most common of enlarged glands. They lie outside the salivary gland superficial to the mylo-hyoid and upon the layer of deep fascia stretching across from the sterno-mastoid to the hyo-glossus, mylo-hyoid, digastric and hyoid bone. In their removal it is difficult for the inferior labial branch of the facial nerve to escape division, the facial vessels are deep to the glands, and can generally be avoided. When much enlarged, their adhesion to the fascia is so intimate as to make them practically continuous with the deep set, and they can then be easily removed in one group. The importance of this group and the frequency of their infection is readily understood when their connexions are considered. They receive lymphatic vessels from the superficial part of the nose, face, and both lips, while the floor of the mouth, front of the tongue, submaxillary and sublingual salivary glands are furnished with vessels that drain into the deeper submaxillary groups under the fascia, so that the intimate connexion of the two groups, when enlarged, is due as much to their simultaneous infection from neighbouring areas as to their direct anatomical continuity.

3. The superficial cervical set includes the glands that run along the external jugular vein superficial to the sternomastoid and along its posterior border, where they lie embedded in the cervical fascia, so that their free removal, in the rare event of the deep glands not being enlarged, exposes the muscular floor of the posterior triangle; the spinal accessory nerve, leaving the posterior border of the muscle a little below its centre, lies beneath the superficial group, and is seldom endangered by their removal, but the descending acromial and clavicular branches of the cervical plexus are usually found running directly over the glands and are not easily avoided.

The deep cervical glands have been divided for descriptive purposes into an upper and a lower set, but no such division is recognizable where most are affected by tuberculous disease. They extend in varying degrees of enlargement from the transverse process of the axis and base of the skull above to the junction of the internal jugular and subclavian veins below, where they pass outwards along the upper edge of the subclavian vein towards the axillary group, and inwards behind the clavicle and sterno-clavicular joints in close relar tion with the innominate vessels and the pleura.

The glands when enlarging tend, like the spread of a deep. seated abscess, to follow well-defined planes of least resistance, and are curiously directed and restrained by the firmer bands into which the cervical fascia is divided. For instance, it is very rare to find enlargement of the group in the anterior triangle to extend lower down along the anterior edge of the sterno-mastoid than the lower border of the thyroid cartilage; below that level they are directed backwards under the muscle over the carotid sheath and bulge out under the posterior border of the muscle, coming thus in contact with. the glandular concatenatae proper that lie directly upon the fascia forming the sheath of the internal jugular vein. This backward direction of the enlarging glands is brought about, no doubt, by the strong pressure exerted on them by the thicker sternal head of the muscle and its intimate fascial connexions, as, where, owing to division of its nerve, the muscle has undergone atrophy, I have several times removed enlarged glands from beneath its anterior border nearly as far down as the clavicle.

In the same way, I have never found any enlarged glands in the closely-confined space above and internal to the posterior belly of the digastric, while outside and above the posterior edge of the digastric and stylo-hyoid their enlargement in cases of any extent is a fairly constant feature.

In notes of my own cases I am accustomed to refer to the deep glands as belonging to three main groups-an anterior group comprising all glands that can be reached from the ront of the sterno-mastoid, and an upper and lower posterior group, these being separated by the spinal accessory nerve passing across the posterior triangle.

The lower posterior group comprises three fairly definite sets: (1) Those glands that were originally superficial or internal to the vessels and have in their growth been directed outwards and backwards in the direction of least resistance : (2) those that belong naturally to the posterior triangle; and (3) those that are in actual contact with the venous compartment of the carotid sheath. In removing these the vein should be deliberately exposed and kept in sight, and is 